\title{
A Pilot Study Comparing Teletherapy with the Conventional Face-to-Face Therapy for Speech-Language Disorders
}

\author{
Tanvi Chaudhary ${ }^{1}$ - Anupam Kanodia ${ }^{1} \cdot$ Hitesh Verma ${ }^{1}$ Chirom Amit Singh ${ }^{1}$. \\ Ashwani Kumar Mishra ${ }^{2} \cdot$ Kapil Sikka $^{1}$ (B)
}

Received: 7 April 2021/ Accepted: 17 May 2021/Published online: 28 May 2021

(C) Association of Otolaryngologists of India 2021

\begin{abstract}
Speech-language therapists along with affected individuals face various challenges for accomplishing the rehabilitation services. In the current COVID19 pandemic scenario, telerehabilitation has emerged as a substitute to the traditional face-to-face therapy, and is the only option possible in some cases. To subjectively assess the feasibility \& acceptability of telerehabilitation provided by speech-language pathologist to patients of speech and language disorders. This qualitative study includes 20 patients suffering from disorders of fluency, voice, swallowing and neurogenic disorders. The participants included were undergoing face-to-face therapy at our institute. After the completion of face-to-face session series, telerehabilitation services were provided through a video calling app. The outcomes of teletherapy were assessed subjectively using a structured questionnaire on 11 parameters using a
\end{abstract}

Kapil Sikka

kapil_sikka@yahoo.com

Tanvi Chaudhary

tanvi.aslp@rediffmail.com

Anupam Kanodia

kanodiaanupam@gmail.com

Hitesh Verma

drhitesh10@gmail.com

Chirom Amit Singh

amitchirom@gmail.com

Ashwani Kumar Mishra

akmaiims@gmail.com

1 Department of Otorhinolaryngology and Head and Neck Surgery, All India Institute of Medical Sciences, New Delhi 110029, India

2 Department of Biostatistics, All India Institute of Medical Sciences, New Delhi India
Likert scale. Of the included 20 patients, after completion of teletherapy, four patients chose the physical interaction as the preferred mode of therapy while 16 chose teletherapy as the preferred mode. Except three clients who rated their overall satisfaction as ' 3 ', others rated as ' 4 ' or ' 5 '.The therapists were satisfied with the outcomes in 17 cases, and were pleased with the overall progress of all the clients (rated 4 or 5). Telerehabilitation is a reliable method to deliver speech and language services at community level, on long-term basis, as is proven by the high satisfaction scores among the clients as well as the service providers.

Clinical Trial Registration The trial has been registered in Clinical Trials Registry of India (CTRI) vide number CTRI/2018/04/ 012,922 (http://ctri.nic.in/Clinicaltrials/log in.php) on 02/04/2018.

Keywords Telepractice $\cdot$ Face-to-face therapy

Teletherapy · Telerehabilitation · Teleservice

\section{Introduction}

Communication disorders have a significant impact on an individual's quality of life and on that of their families [1]. Such patients require regular stimulation through speech therapy on a long term basis. In a country like India, this often means travel across long distances, loss of wages for the family and limited access to treating experts. These hassles have been exaggerated in the current COVID-19 pandemic scenario where means to travel are limited. The Rehabilitation Council of India endorsed teletherapy for communication disorders in 2015 [2]. This has prompted us to explore teletherapy as a possible source of rehabilitation and follow-up. 


\section{Materials and Methods}

A prospective, comparative study was conducted from March to August 2018 at our tertiary care center. Ethical clearance was obtained (RP-40/2018). The trial was registered at Clinical Trials Registry of India (CTRI) vide number CTRI/2018/04/ 012,922. Twenty patients with disorders of fluency, voice, adult neurogenic and swallowing (5 participants in each category) who got enrolled in RUAS (Rehabilitation Unit of Audiology and Speechlanguage pathology), department of ENT for speech and language therapy or those who were already receiving faceto-face therapy sessions at our institute were considered for inclusion. Written consent was obtained from all the participants. Patients aged $<15$ years, those with a previously persisting speech/language/voice/swallowing disorder, having poor vision or hearing, those with attention deficit, bilateral motor impairment, severely impaired comprehension, those suspected of malingering, not speaking the native Hindi language and the patients who did not possess a smart phone or a computer were excluded from the study. A detailed clinical history was obtained and all subjects underwent psychological, hearing and vision screening tests for gross abnormalities.

Initially the study participants were provided face-toface therapy sessions, on completion of which, rehabilitation services were provided through telepractice using a video calling app. Each therapy session was conducted for 30 min once a week for a period of 5 weeks in Hindi language. The patient's progress was monitored by the therapist through an informal communication with the participant and his/her family. The level of progress was then used to further customize therapy sessions.

The participants (and care takers where applicable) were given a trial session before initiating telerehabilitation sessions, to guide them the use of video calling app for therapy. The subjects who intended to use therapy services from other centres were excluded from analysis. During the therapy session, to provide supplementary support if necessary, either party could disrupt, momentarily pause and begin a two-way communication using text and/or voice call as appropriate. Participants were instructed to practice the therapy activities each day at home, for a minimum of 30 min with no upper time limit.

A structured questionnaire based on Likert scale (shown in Table 1) was developed to assess various parameters. Content validity was established by a preliminary study on five subjects where none of the participant faced any difficulty in comprehending the statements.

\section{Statistical Analysis}

Statistical analysis was conducted using SPSS version 25.0. Each of the categorical variables was described in terms of frequency and percentage. Qualitative parameters were presented as mean and standard deviation.

\section{Result}

Twenty participants (19 males, one female) with disorders of fluency, voice, swallowing and adult neurogenic disorders (five in each category) were included. Analysis of the parameters asked from the patients and clinician have been summarized in Tables 2 and 3.

From clinician's perspective, positive correlation was observed between face-to-face therapy and teletherapy for progress made by the client and overall satisfaction parameters. However, due to poor connectivity overall satisfaction was rated ' 3 ' for three patients Table 3 .

From client's perspective, subjective evaluation of progress showed comparable scores for both the modes of therapy (all rated either 4 or 5). All participants felt teletherapy was a more convenient, cost effective and comfortable method (rated 4 or 5) of delivering services. When participants were asked questions like 'if teleservice could meet their expectations', 'if they would like to avail teleservices in future' and 'if they would recommend others to take speech therapy through digital media' most of the participants agreed in the affirmative (rating 4 or 5) except one (rating 3 ) who suffered poor connectivity during the teletherapy sessions.

When asked to rate statements concerning overall satisfaction and video quality of teleservice, most answers showed high satisfaction (rated 4 or 5) except three participants (with average satisfaction) for both the parameters who faced connectivity issue during teletherapy services. Moreover, when participants were asked to rate audio quality during teletherapy series, all expressed satisfaction (rated 4 or 5) except four subjects among which two reported average and the other two reported below average audio quality.

Though our results reveal comparable satisfaction and progress for our clients, four of them chose face-to-face therapy over teletherapy at the end of the teletherapy sessions. Seven of the clients preferred teletherapy and nine of them found both the modes equally good. 
Table 1 Questionnaire

Please rate the following statements on likert scale ranging from 1 to 5

1 -Not very good/ never / strongly disagree

2 -Below average/ rarely/ somewhat disagree

3- Average/ sometimes/ no opinion

4- Above average/ often/ somewhat agree

5 -Very good / always/ strongly agree

Participant perspective

1. Audio quality in telerehabilitation method

2. Video quality in telerehabilitation method

3. Level of comfort with telerehabilitation method

4. Cost-effectiveness of telerehabilitation method

5. Convenience in accessing services with telerehabilitation method

6. Progress with telerehabilitation method

7. Telerehabilitation method met the expectations

8. Likelihood of future participation in telerehabilitation sessions

9. Likelihood of recommending telerehabilitation method to someone else

10. Preference for interacting with clinician with telerehabilitation method

11. Overall satisfaction with telerehabilitation method

Other issue(s)-

Clinician perspective

1. Progress with telerehabilitation method

2. Overall satisfaction with telerehabilitation method

Table 2 Mean (standard deviation) of all the qualitative parameters asked from clients and the clinician

\begin{tabular}{llr}
\hline Perspective & Parameters & Mean (SD) \\
\hline Client & Audio quality & $4.05(.945)$ \\
& Video quality & $4.25(.716)$ \\
& Comfort & $4.80(.410)$ \\
& Cost effective & $5.00(.000)$ \\
& Convenience & $5.00(.000)$ \\
& Progress & $4.85(.366)$ \\
& Expectations met & $4.70(.571)$ \\
& Future participation & $4.70(.571)$ \\
& Recommend others & $4.75(.550)$ \\
Clinician & Preference of interacting with clinician & $3.20(.834)$ \\
& Overall satisfaction & $4.30(.733)$ \\
& Progress & $4.85(.366)$ \\
& Overall satisfaction & $4.30(.733)$
\end{tabular}

\section{Discussion}

The present study sought to evaluate teletherapy as a viable alternative to conventional face-to-face therapy. The results of teletherapy were determined through a questionnaire, answered by the therapist and the patient. Our results show that teletherapy is a comfortable, cheap and convenient method to deliver speech and swallowing therapy sessions on a long-term basis. The previous studies by Mashima et al. [3], Theodores et al. [4] have documented similar findings where they recognized significant progress in the participants who were suffering from voice and speech disorders (Parkinson's disease) respectively. 
Table 3 Number (percentage) of participants who endorsed specific ratings on questions related to teletherapy

\begin{tabular}{|c|c|c|c|c|c|c|}
\hline Perspective & Parameters & Rated 5 & Rated 4 & Rated 3 & Rated 2 & Rated 1 \\
\hline \multirow[t]{11}{*}{ Client } & Audio quality & $7(35 \%)$ & $9(45 \%)$ & $2(10 \%)$ & $2(10 \%)$ & 0 \\
\hline & Video quality & $8(40 \%)$ & $9(45 \%)$ & $2(10 \%)$ & 0 & 0 \\
\hline & Comfort & $16(80 \%)$ & $4(20 \%)$ & 0 & 0 & 0 \\
\hline & cost effective & $20(100 \%)$ & 0 & 0 & 0 & 0 \\
\hline & Convenience & $20(100 \%)$ & 0 & 0 & 0 & 0 \\
\hline & Progress & $17(85 \%)$ & $3(15 \%)$ & 0 & 0 & 0 \\
\hline & Expectations met & $16(80 \%)$ & $4(20 \%)$ & 0 & 0 & 0 \\
\hline & Future participation & $15(75 \%)$ & $4(20 \%)$ & $1(5 \%)$ & 0 & 0 \\
\hline & Recommend others & $16(80 \%)$ & $3(15 \%)$ & $1(5 \%)$ & 0 & 0 \\
\hline & Preference of interacting with clinician & $1(5 \%)$ & $6(30 \%)$ & $9(45 \%)$ & $4(20 \%)$ & 0 \\
\hline & Overall satisfaction & $9(45 \%)$ & $8(40 \%)$ & $3(15 \%)$ & 0 & 0 \\
\hline \multirow[t]{2}{*}{ Clinician } & Progress & $17(85 \%)$ & $3(15 \%)$ & 0 & 0 & 0 \\
\hline & Overall satisfaction & $9(45 \%)$ & $8(40 \%)$ & $3(15 \%)$ & 0 & 0 \\
\hline
\end{tabular}

The current study shows that telerehabilitation is a dependable and effective mode of service provision. The participants and clinicians in our study have reported above average levels of satisfaction and progress with telepractice service, Grogan-Johnson et al. [5] in a pilot study on school-going children have reported excellent satisfaction and progress results from the therapists as well as the parents when teletherapy and face-to-face therapy were delivered in sessions of four months each. ScheidemanMiller et al. [6], reported improved problem-solving capacities, memory and social interaction skills among school children when teletherapy was provided over a 5-week period.

Tucker et al. [7] cite technological failure as the primary barrier that prevents effective delivery of teletherapy. Isaki and Farrell [8] in their study reported that video and audio transmission quality was a concern for $60 \%$ of the participants. In our study, four participants raised issues with audio quality and only two out of twenty participants raised concern over the video quality. This underscores the importance of an uninterrupted technology in providing teletherapy and how frequent interruptions lead to poor satisfaction scores, both from the patient and the clinician side. We thus, recommend consideration of teletherapy for patients who are digitally literate and have access to uninterrupted communication services. Patients with the above characteristics who live remotely and have limited access to speech-language therapists are more likely to report it as convenient [9] and cost-effective [10] method of delivering services as pointed by different authors. Our study also recognized teletherapy to be convenient and cost-effective method with all the participants giving highest rating. In the present study, $9(45 \%)$ participants had no preference for either mode of treatment and 7 (35\%) preferred teletherapy as the preferred mode. Fridler et al. [11] report good agreement between the two modes of treatment in terms of comfort, recommending others, future participation and expectations being met. However, $58 \%$ of their subjects had no preference to either mode and $10 \%$ chose teletherapy. A higher population choosing teletherapy in our study may be attributed to the fact that it is a cost-effective measure for many of our patients, and cost of treatment is a prime concern to the population our hospital caters to. Also, Tucker [7] has discussed other barriers of teleservices in communication disorders. As per the author, loss of privacy and confidentiality and inadequate training of the client as well as the service-providers were the major limitations while embarking on such a project, however, it did improve access to remote areas and saved considerable expense from the patient side. We agree that ensuring confidentiality and privacy is a challenge while using an internet based platform to deliver our services. It is necessary that the service-provider and the recipient are adequately trained to handle such technology to ensure smooth functioning and prevent loss of personal data.

Our study reveals that in carefully selected patient groups, teletherapy can achieve progress and satisfaction scores at par with the conventional face-to-face therapy, and in some others it can be used to augment the results in combination with conventional face-to-face therapy. Though, the study has few limitations. It's a non-randomized controlled trial with small sample size. Also, formal training for clinician and clients along with formal evaluation methods could have provided more appropriate results. 


\section{Conclusion}

Face-to-face therapies have always been considered as 'gold standard' for care of patients with speech, language and swallowing disorders, though teletherapy has emerged as a viable alternative for patients with a decent educational and technological background and should be evaluated by studies of larger sample size.

\section{Acknowledgements Nil.}

Funding The study was not supported by any funding agency.

Data Availability All data and material are available in institute's record.

\section{Declarations}

Conflict of interest The authors have no conflicts of interest to declare that are relevant to the content of the article.

Consent to Participate The written informed consent was taken from each subject. The identity of subjects was not disclosed. Informed consent was obtained from all individual participants included in the study.

Consent for Publication All the authors have read and approved the paper for publication. The authors affirm that human research participants provided informed consent for publication of the data.

Ethical Approval The study was conducted after receiving approval from AIIMS, New Delhi Institutional Ethics Committee.

Human or Animal Rights All procedures performed in study were in accordance with the ethical standards of the institutional and/or national research committee and with the 1964 Helsinki declaration and its later amendments or comparable ethical standard.

\section{References}

1. Clegg J, Hollis C, Mawhood L, Rutter M (2005) Developmental language disorders-a follow-up in later adult life. Cognitive, language and psychosocial outcomes. J Child Psychol Psychiatry Engl 46:128-49

2. Rangaswamy Y, Rao P (2018) Tele speech-language pathology and audiology in India - a short report. J Int Soc Telemed eHealth 6:e19-1

3. Mashima PA, Birkmire-Peters DP, Syms MJ, Holtel MR, Burgess LPA, Peters LJ (2003) Telehealth: voice therapy using telecommunications technology. Am J Speech Lang Pathol 12:432-439

4. Theodoros DG, Constantinescu G, Russell TG, Ward EC, Wilson SJ, Wootton R (2006) Treating the speech disorder in parkinson's disease online. J Telemed Telecare 12:88-91

5. Grogan-Johnson S, Alvares R, Rowan L, Creaghead N (2010) A pilot study comparing the effectiveness of speech language therapy provided by telemedicine with conventional on-site therapy. J Telemed Telecare Engl 16:134-139

6. Scheideman-Miller C, Clark P, Smeltzer S, Carpenter J, Hodge B, Proutry D (2002) Two year results of a pilot study delivering speech therapy to children in a rural Oklahoma school via telemedicine. In: Proceedings of the 35th annual Hawaii international conference on system sciences (HICSS'02), IEEE computer society, vol 6, USA, p 161.2

7. Tucker JK (2012) Perspectives of speech-language pathologists on the use of telepractice in schools: the qualitative view. Int $\mathrm{J}$ Telerehabilit 4:47-60

8. Isaki E, Fangman Farrell C (2015) Provision of speech-language pathology telepractice services using apple iPads. Telemed $\mathbf{J}$ e-health Off J Am Telemed Assoc U S 21:538-49

9. Burgoyne N, Cohn AN (2020) Lessons from the transition to relational teletherapy during covid-19. Fam Proc 59(3):974-988

10. Tsou C, Robinson S, Boyd J et al (2020) Effectiveness and costeffectiveness of telehealth in rural and remote emergency departments: a systematic review protocol. Syst Rev 9:82

11. Fridler N, Rosen K, Menahemi-Falkov M, Herzberg O, Lev A, Kaplan D, et al. (2012) Tele-rehabilitation therapy vs. face-toface therapy for aphasic patients. In: eTELEMED 2012: the fourth international conference on eHealth, telemedicine, and social medicine, pp.18-23. https://www.researchgate.net/publica tion/274713926_Tele-Rehabilitation_Therapy_vs_Face-to-

Face_Therapy_for_Aphasic_Patients. Accessed 16 May 2020

Publisher's Note Springer Nature remains neutral with regard to jurisdictional claims in published maps and institutional affiliations. 